\title{
EXISTENCE AND NON-EXISTENCE OF FREQUENTLY HYPERCYCLIC SUBSPACES FOR WEIGHTED SHIFTS
}

\author{
QUENTIN MENET \\ (Communicated by Pamela B. Gorkin)
}

\begin{abstract}
We study the existence and the non-existence of frequently hypercyclic subspaces of frequently hypercyclic operators living on Banach spaces. In particular, we give an example of a weighted shift on $l^{p}$ possessing a frequently hypercyclic subspace and an example of a frequently hypercyclic weighted shift on $l^{p}$ possessing a hypercyclic subspace but no frequently hypercyclic subspace. The latter example allows us to answer positively Problem 1 posed by Bonilla and Grosse-Erdmann in [Monatsh. Math. 168 (2012)].
\end{abstract}

\section{INTRODUCTION}

Let $X$ be a separable infinite-dimensional Banach space. We denote by $L(X)$ the space of continuous linear operators on $X$.

An operator $T \in L(X)$ is said to be hypercyclic if there exists a vector $x \in X$ (called hypercyclic) such that the set $\left\{T^{n} x: n \geq 0\right\}$ is dense in $X$. The hypercyclicity is an important notion of linear dynamics and we refer to the books [3,7] for more details about this theory. In 2004, Bayart and Grivaux [1,2] defined a new notion of hypercyclicity, called frequent hypercyclicity. An operator $T \in L(X)$ is said to be frequently hypercyclic if there exists a vector $x \in X$ (also called frequently hypercyclic) such that for any non-empty open set $U \subset X$, $\underline{\operatorname{dens}}\left\{n \geq 0: T^{n} x \in U\right\}>0$, where for any subset $A$ of $\mathbb{Z}_{+}$(the set of non-negative integers), we define the lower density of $A$ as

$$
\underline{\operatorname{dens}} A=\liminf _{N \rightarrow \infty} \frac{\#(A \cap[0, N])}{N+1} .
$$

A useful sufficient condition for frequent hypercyclicity has been given by Bayart and Grivaux [1,2] and improved by Bonilla and Grosse-Erdmann [5]:

Theorem 1.1 (Frequent Hypercyclicity Criterion). Let $X$ be a separable infinitedimensional Banach space and $T \in L(X)$. Suppose that there exist a dense subset $X_{0}$ of $X$ and a map $S: X_{0} \rightarrow X_{0}$ such that, for all $x \in X_{0}$,

(1) $\sum_{n=1}^{\infty} T^{n} x$ converges unconditionally,

(2) $\sum_{n=1}^{\infty} S^{n} x$ converges unconditionally,

(3) $T S x=x$.

Then $T$ is frequently hypercyclic.

Received by the editors September 30, 2013, and, in revised form, January 7, 2014.

2010 Mathematics Subject Classification. Primary 47A16.

Key words and phrases. Hypercyclicity, frequent hypercyclicity, hypercyclic subspaces, weighted shifts.

The author was supported by a grant of FRIA. 
In this paper, we are interested in the existence of infinite-dimensional closed subspaces in which every non-zero vector is frequently hypercyclic. Such a subspace is called a frequently hypercyclic subspace. The notion of a frequently hypercyclic subspace was studied for the first time by Bonilla and Grosse-Erdmann [6] . In particular, they obtained the following sufficient condition for the existence of frequently hypercyclic subspaces:

Theorem $1.2(\underline{6})$. Let $X$ be a separable infinite-dimensional Banach space and $T \in L(X)$. Suppose that

(1) T satisfies the Frequent Hypercyclicity Criterion;

(2) there exists an infinite-dimensional closed subspace $M_{0}$ of $X$ such that, for all $x \in M_{0}, T^{n} x \rightarrow 0$ as $n \rightarrow \infty$.

Then $T$ has a frequently hypercyclic subspace.

The convergence to 0 along the whole sequence $(n)$ for each vector in $M_{0}$ is a strong assumption and the examples of frequently hypercyclic operators satisfying this criterion are mainly operators for which there exists some scalar $\lambda$ with $|\lambda|<1$ such that $\operatorname{dim} \operatorname{ker}(T-\lambda)=\infty$. In particular, so far no weighted shift with frequently hypercyclic subspaces is known.

On the other hand, an operator $T$ possesses a hypercyclic subspace if there exists an infinite-dimensional closed subspace in which every non-zero vector is hypercyclic for $T$. Obviously, if $T$ does not possess a hypercyclic subspace or if $T$ is not frequently hypercyclic, then $T$ does not possess a frequently hypercyclic subspace. Bonilla and Grosse-Erdmann then asked the following question [6, Problem 1]:

Problem 1. Does there exist a frequently hypercyclic operator that has a hypercyclic subspace but not a frequently hypercyclic subspace?

In Section 2, we state a sufficient condition for the non-existence of frequently hypercyclic subspaces. Then we show that there exists some frequently hypercyclic weighted shift on $l^{p}$ that possesses a hypercyclic subspace and that satisfies this condition. This allows us to answer Problem 1 positively.

In Section 3, we state a slight improvement of Theorem 1.2 and we give an example of a weighted shift on $l^{p}$ possessing a frequently hypercyclic subspace.

\section{Non-EXISTENCE OF FREQUENTLY HYPERCYCLIC SUBSPACES}

In the case of hypercyclic subspaces, we know a simple criterion 9,10 , such that if an operator $T$ satisfies this criterion, then $T$ does not possess any hypercyclic subspace.

Theorem 2.1. Let $X$ be a separable infinite-dimensional Banach space and $T$ $\in L(X)$. If there exist $C>1$, a closed subspace $E$ of finite codimension in $X$ and a positive integer $n \geq 1$ such that

$$
\left\|T^{n} x\right\| \geq C\|x\| \quad \text { for any } x \in E,
$$

then $T$ does not possess any hypercyclic subspace.

In fact, one can show that if $T$ satisfies the assumption of Theorem 2.1, then each infinite-dimensional closed subspace $M$ of $X$ contains a vector $x$ such that $\left\|T^{n} x\right\| \geq 1$ for any $n$. The construction of such a vector $x$ relies on the following lemma. 
Lemma 2.2 ([7, Lemma 10.39]). Let $X$ be a separable infinite-dimensional Banach space. For any finite-dimensional subspace $F$ of $X$, any $\varepsilon>0$, there exists a closed subspace $E$ of finite codimension in $X$ such that for any $x \in E$ and any $y \in F$,

$$
\|x+y\| \geq \max \left(\frac{\|x\|}{2+\varepsilon}, \frac{\|y\|}{1+\varepsilon}\right) .
$$

We would like a similar criterion for the notion of frequently hypercyclic subspaces. Obviously, if $T$ does not possess any hypercyclic subspace, then $T$ does not possess any frequently hypercyclic subspace. Nevertheless, in order to prove that an operator does not possess any frequently hypercyclic subspace, it is sufficient to prove that each infinite-dimensional closed subspace $M$ contains a vector $x$ such that the upper density of $\left\{n \geq 0:\left\|T^{n} x\right\| \geq C\right\}$ is equal to 1 for some $C>0$, i.e.

$$
\limsup _{N \rightarrow \infty} \frac{\#\left\{n \leq N:\left\|T^{n} x\right\| \geq C\right\}}{N+1}=1 .
$$

Indeed, such a vector $x$ cannot be frequently hypercyclic since the lower density of $\left\{n \geq 0:\left\|T^{n} x\right\|<C\right\}$ would be equal to 0 .

Theorem 2.3. Let $X$ be a separable infinite-dimensional Banach space and $T$ $\in L(X)$. If there exists $C>0$ such that for any $K \geq 1$, any infinite-dimensional closed subspace $M$ of $X$, any $x \in X$, there exists $x^{\prime} \in M$ such that $\left\|x^{\prime}\right\| \leq \frac{1}{K}$ and

$$
\sup _{k>K} \frac{\#\left\{n \leq k:\left\|T^{n}\left(x+x^{\prime}\right)\right\| \geq C\right\}}{k+1}>1-\frac{1}{K},
$$

then $T$ does not possess any frequently hypercyclic subspace.

Proof. Let $\tilde{M}$ be an infinite-dimensional closed subspace of $X$. We show that there exists a vector $x \in \tilde{M}$ such that

$$
\limsup _{N \rightarrow \infty} \frac{\#\left\{n \leq N:\left\|T^{n} x\right\| \geq \frac{C}{2}\right\}}{N+1}=1 .
$$

To this end, we recursively construct a sequence $\left(x_{n}\right)_{n \geq 0} \subset \tilde{M}$ with $x_{0}=0$ and an increasing sequence $\left(k_{n}\right)_{n \geq 0}$ with $k_{0}=0$ such that for any $n \geq 1$,

(1) $\left\|x_{n}\right\| \leq \frac{1}{n^{2}}$;

(2) we have

$$
\frac{\#\left\{j \leq k_{n}:\left\|T^{j}\left(\sum_{k=1}^{n} x_{k}\right)\right\| \geq C\right\}}{k_{n}+1}>1-\frac{1}{n^{2}}
$$

(3) for any $j \leq k_{n-1}, T^{j} x_{n} \in \bigcap_{k<n-1} E_{k, j}$, where $E_{k, j}$ is the closed subspace of finite codimension given by Lemma 2.2 for $F_{k, j}=\operatorname{span}\left(T^{j} x_{0}, \ldots, T^{j} x_{k}\right)$ and $\varepsilon=1$.

At each step, we obtain such a vector $x_{n}$ by using our assumption for $K=$ $\max \left\{n^{2}, k_{n-1}\right\}, M=\tilde{M} \cap \bigcap_{j \leq k_{n-1}} T^{-j}\left(\bigcap_{k \leq n-1} E_{k, j}\right)$ and $x=\sum_{k=1}^{n-1} x_{k}$. 
We let $x:=\sum_{n=1}^{\infty} x_{n}$ and $J_{n}=\left\{j \leq k_{n}:\left\|T^{j}\left(\sum_{k=1}^{n} x_{k}\right)\right\| \geq C\right\}$. We know that $x \in \tilde{M}$ and moreover, for any $n \geq 1$, any $j \in J_{n}$, we have

$$
\begin{aligned}
\left\|T^{j} x\right\| & =\left\|\sum_{k=1}^{\infty} T^{j} x_{k}\right\| \\
& \geq \frac{1}{2}\left\|\sum_{k=1}^{n} T^{j} x_{k}\right\| \quad \text { because } \sum_{k=n+1}^{\infty} T^{j} x_{k} \in E_{n, j} \text { and } \sum_{k=1}^{n} T^{j} x_{k} \in F_{n, j} \\
& \geq \frac{C}{2} \quad \text { because } j \in J_{n} .
\end{aligned}
$$

We deduce that for any $n \geq 1$,

$$
\frac{\#\left\{j \leq k_{n}:\left\|T^{j} x\right\| \geq \frac{C}{2}\right\}}{k_{n}+1} \geq \frac{\# J_{n}}{k_{n}+1} \geq 1-\frac{1}{n^{2}} \underset{n \rightarrow \infty}{\longrightarrow} 1 .
$$

The result follows.

In the case of weighted shifts, Theorem 2.3 gives us a simple sufficient condition for the non-existence of frequently hypercyclic subspaces. We recall that a weighted shift $B_{w}: l^{p} \rightarrow l^{p}$ is defined by

$$
B_{w}\left(\left(x_{0}, x_{1}, x_{2}, \ldots\right)\right)=\left(w_{1} x_{1}, w_{2} x_{2}, w_{3} x_{3}, \ldots\right),
$$

where $w=\left(w_{n}\right)_{n \geq 1}$ is a bounded sequence of non-zero scalars. In other words, we have $B_{w} e_{k}=w_{k} e_{k-1}$, where $\left(e_{k}\right)_{k \geq 0}$ is the canonical basis of $l^{p}$ and $e_{-1}=0$.

Theorem 2.4. Let $B_{w}: l^{p} \rightarrow l^{p}$ be a weighted shift $(1 \leq p<\infty)$. If there exists a sequence $\left(C_{k}\right)_{k \geq 0}$ of positive numbers such that $\sum_{k \geq 0}\left(1 / C_{k}\right)^{p}<\infty$ and

$$
\frac{\#\left\{n \geq 0:\left\|B_{w}^{n} e_{k}\right\| \geq C_{k}\right\}}{k+1} \underset{k \rightarrow \infty}{\longrightarrow} 1,
$$

then $B_{w}$ does not possess any frequently hypercyclic subspace.

Proof. Let $K \geq 1, M$ be an infinite-dimensional closed subspace of $l^{p}$ and $x \in l^{p}$. In view of Theorem [2.3, it suffices to show that there exists $x^{\prime} \in M$ such that $\left\|x^{\prime}\right\| \leq \frac{1}{K}$ and

$$
\sup _{k>K} \frac{\#\left\{n \leq k:\left\|B_{w}^{n}\left(x+x^{\prime}\right)\right\| \geq 1\right\}}{k+1}>1-\frac{1}{K} .
$$

We first remark that for any $x^{\prime} \in l^{p}$, any $k \geq 0$,

$$
\frac{\#\left\{n \leq k:\left\|B_{w}^{n}\left(x+x^{\prime}\right)\right\| \geq 1\right\}}{k+1} \geq \frac{\#\left\{n \geq 0:\left\|\left(x_{k}+x_{k}^{\prime}\right) B_{w}^{n} e_{k}\right\| \geq 1\right\}}{k+1}
$$

and thus, if $\left|x_{k}+x_{k}^{\prime}\right| \geq 1 / C_{k}$, we have

$$
\frac{\#\left\{n \leq k:\left\|B_{w}^{n}\left(x+x^{\prime}\right)\right\| \geq 1\right\}}{k+1} \geq \frac{\#\left\{n \geq 0:\left\|B_{w}^{n} e_{k}\right\| \geq C_{k}\right\}}{k+1} .
$$

By (2.1), we know that there exists $k_{0}$ such that for any $k \geq k_{0}$

$$
\frac{\#\left\{n \geq 0:\left\|B_{w}^{n} e_{k}\right\| \geq C_{k}\right\}}{k+1}>1-\frac{1}{K} .
$$


We deduce that it suffices to find a vector $x^{\prime} \in M$ such that $\left\|x^{\prime}\right\| \leq \frac{1}{K}$ and $\left|x_{k}+x_{k}^{\prime}\right| \geq 1 / C_{k}$ for some $k \geq \max \left(k_{0}, K+1\right)$. Let $k_{1} \geq \max \left(k_{0}, K+1\right)$ such that

$$
\left(\sum_{k=k_{1}}^{\infty}\left|x_{k}\right|^{p}\right)^{\frac{1}{p}} \leq \frac{1}{2 K} \text { and }\left(\sum_{k=k_{1}}^{\infty} \frac{1}{C_{k}^{p}}\right)^{1 / p} \leq \frac{1}{2 K} .
$$

Since $M$ is an infinite-dimensional closed subspace, we know that for any $k \geq 0, M$ contains a vector $y$ with valuation $v(y):=\inf \left\{j \geq 0: y_{j} \neq 0\right\}$ larger than $k$. We can thus choose $x^{\prime} \in M$ such that

$$
v\left(x^{\prime}\right) \geq k_{1} \quad \text { and } \quad\left\|x^{\prime}\right\|=1 / K .
$$

Using (2.2), we deduce that

$$
\begin{aligned}
\left(\sum_{k=k_{1}}^{\infty}\left|x_{k}^{\prime}+x_{k}\right|^{p}\right)^{1 / p} & \geq\left\|x^{\prime}\right\|-\left(\sum_{k=k_{1}}^{\infty}\left|x_{k}\right|^{p}\right)^{1 / p} \\
& \geq \frac{1}{K}-\frac{1}{2 K}=\frac{1}{2 K} \\
& \geq\left(\sum_{k=k_{1}}^{\infty} \frac{1}{C_{k}^{p}}\right)^{1 / p}
\end{aligned}
$$

and thus, there exists $k \geq k_{1}$ such that

$$
\left|x_{k}^{\prime}+x_{k}\right| \geq \frac{1}{C_{k}}
$$

which concludes the proof.

We can now prove the existence of a frequently hypercyclic weighted shift possessing a hypercyclic subspace and no frequently hypercyclic subspace. Thanks to Bayart and Ruzsa 4, we know a characterization of frequently hypercyclic weighted shifts on $l^{p}$. A weighted shift $B_{w}: l^{p} \rightarrow l^{p}$ is frequently hypercyclic if and only if $B_{w}$ satisfies the Frequent Hypercyclicity Criterion, and if and only if

$$
\sum_{k=1}^{\infty} \frac{1}{\prod_{\nu=1}^{k}\left|w_{\nu}\right|^{p}}<\infty
$$

We also know a characterization of weighted shifts on $l^{p}$ with hypercyclic subspaces [8, 10]. A hypercyclic weighted shift $B_{w}: l^{p} \rightarrow l^{p}$ possesses a hypercyclic subspace if and only if

$$
\sup _{n \geq 1} \inf _{k \geq 0} \prod_{\nu=1}^{n}\left|w_{k+\nu}\right| \leq 1 .
$$

In conclusion, it just remains to show that there is some bounded weighted sequence $w$ satisfying (2.3), (2.4) and the condition of Theorem 2.4

Theorem 2.5. There exists some frequently hypercyclic weighted shift on $l^{p}$ possessing a hypercyclic subspace and no frequently hypercyclic subspace.

Proof. Let $\left(a_{n}\right)_{n \geq 0}$ be a strictly increasing sequence of integers such that $a_{0}=1$. We consider the weighted sequence $w$ given by $w_{k}=2$ if $k \in\left[a_{2 n}, a_{2 n+1}\right.$ [ for some $n \geq 0$, and $w_{k}=1$ otherwise. 
Since the sequence $w$ is bounded, the weighted shift $B_{w}$ is an operator on $l^{p}$. We first show that if

$$
\sum_{n=0}^{\infty} \frac{a_{2 n+2}-a_{2 n+1}}{\left(2^{a_{2 n+1}-a_{2 n}}\right)^{p}}<\infty,
$$

then $B_{w}$ is frequently hypercyclic. We remark that for any $k \geq a_{2 n+1}$, we have $\prod_{\nu=1}^{k}\left|w_{\nu}\right| \geq 2^{a_{2 n+1}-a_{2 n}}$ and if $\left(n_{j}\right)_{j \geq 1}$ is the increasing enumeration of the set $\cup_{n=0}^{\infty}\left[a_{2 n}, a_{2 n+1}\left[\right.\right.$, then $\prod_{\nu=1}^{n_{j}}\left|w_{\nu}\right|=2^{j}$. We therefore deduce that

$$
\begin{aligned}
\sum_{k=1}^{\infty} \frac{1}{\prod_{\nu=1}^{k}\left|w_{\nu}\right|^{p}} & =\sum_{n=0}^{\infty}\left(\sum_{k \in\left[a_{2 n}, a_{2 n+1}[\right.} \frac{1}{\prod_{\nu=1}^{k}\left|w_{\nu}\right|^{p}}+\sum_{k \in\left[a_{2 n+1}, a_{2 n+2}[\right.} \frac{1}{\prod_{\nu=1}^{k}\left|w_{\nu}\right|^{p}}\right) \\
& =\sum_{j=1}^{\infty} \frac{1}{\prod_{\nu=1}^{n_{j}}\left|w_{\nu}\right|^{p}}+\sum_{n=0}^{\infty} \sum_{k \in\left[a_{2 n+1}, a_{2 n+2}[\right.} \frac{1}{\prod_{\nu=1}^{k}\left|w_{\nu}\right|^{p}} \\
& \leq \sum_{j=1}^{\infty}\left(\frac{1}{2^{j}}\right)^{p}+\sum_{n=0}^{\infty} \frac{a_{2 n+2}-a_{2 n+1}}{\left(2^{\left.a_{2 n+1}-a_{2 n}\right)^{p}}\right.}
\end{aligned}
$$

and, in view of (2.3), we conclude that if

$$
\sum_{n=0}^{\infty} \frac{a_{2 n+2}-a_{2 n+1}}{\left(2^{a_{2 n+1}-a_{2 n}}\right)^{p}}<\infty,
$$

then $B_{w}$ is frequently hypercyclic.

Thanks to (2.4), we also know that if $a_{2 n+2}-a_{2 n+1}$ tends to infinity, then $B_{w}$ possesses a hypercyclic subspace and, finally, we remark that we have, for any $l \geq a_{2 n+1}$,

$$
\#\left\{k \geq 0:\left\|B_{w}^{k} e_{l}\right\| \geq 2^{a_{2 n+1}-a_{2 n}}\right\} \geq a_{2 n} .
$$

Thus, if for any $a_{2 n+1} \leq l<a_{2 n+3}$, we let $C_{l}=2^{a_{2 n+1}-a_{2 n}}$, then we have

$$
\frac{\#\left\{k \geq 0:\left\|B_{w}^{k} e_{l}\right\| \geq C_{l}\right\}}{l+1} \geq \frac{a_{2 n}}{a_{2 n+3}} \quad \text { and } \quad \sum_{l=a_{1}}^{\infty} \frac{1}{C_{l}^{p}}=\sum_{n=0}^{\infty} \frac{a_{2 n+3}-a_{2 n+1}}{\left(2^{a_{2 n+1}-a_{2 n}}\right)^{p}} .
$$

Therefore, if

$$
\sum_{n=0}^{\infty} \frac{a_{2 n+3}-a_{2 n+1}}{\left(2^{a_{2 n+1}-a_{2 n}}\right)^{p}}<\infty \text { and } \frac{a_{2 n}}{a_{2 n+3}} \rightarrow 1,
$$

we deduce from Theorem 2.4 that $B_{w}$ does not possess any frequently hypercyclic subspace.

We now show that if we let

$$
a_{2 n}=1+n(n+1) \quad \text { and } \quad a_{2 n+1}=1+(n+1)^{2},
$$

then each of the properties above is satisfied, which will conclude the proof. We first remark that

$$
a_{2 n+1}-a_{2 n}=n+1=a_{2 n+2}-a_{2 n+1} .
$$

The operator $B_{w}$ is thus frequently hypercyclic because

$$
\sum_{n=0}^{\infty} \frac{a_{2 n+2}-a_{2 n+1}}{\left(2^{a_{2 n+1}-a_{2 n}}\right)^{p}}=\sum_{n=0}^{\infty} \frac{n+1}{\left(2^{n+1}\right)^{p}}<\infty,
$$


and, as $a_{2 n+2}-a_{2 n+1}$ tends to infinity, the operator $B_{w}$ possesses a hypercyclic subspace. Moreover, we remark that

$$
\frac{a_{2 n}}{a_{2 n+3}}=\frac{1+n(n+1)}{1+(n+2)^{2}} \rightarrow 1
$$

and

$$
\sum_{n=0}^{\infty} \frac{a_{2 n+3}-a_{2 n+1}}{\left(2^{a_{2 n+1}-a_{2 n}}\right)^{p}}=\sum_{n=0}^{\infty} \frac{(n+2)^{2}-(n+1)^{2}}{\left(2^{n+1}\right)^{p}}=\sum_{n=0}^{\infty} \frac{2 n+3}{\left(2^{n+1}\right)^{p}}<\infty .
$$

The operator $B_{w}$ is thus a frequently hypercyclic weighted shift possessing a hypercyclic subspace and no frequently hypercyclic subspace.

\section{EXISTENCE OF FREQUENTLY HYPERCYCLIC SUBSPACES}

Let $A$ be a set of positive lower density and $\left(n_{k}\right)$ the increasing enumeration of $A$. It is obvious that if $J$ is a set of positive lower density, then $\left\{n_{k}: k \in J\right\}$ is still a set of positive lower density. Using this argument, it is not difficult to adapt the proof of [6. Lemma 1] in order to force the sets $E(l, j)$ (given by this lemma) to be included in $A$. Indeed, we can see in the proof of this lemma that the sets $E(l, j)$ just have to be sets of positive lower density satisfying convenient separation properties. In view of the previous argument, we can therefore replace the sets $E(l, j)$ by the sets $\left\{n_{k}: k \in E(l, j)\right\} \subset A$. Thanks to this improvement, the proof of Theorem 1.2 can then be adapted in order to obtain the following generalization.

Theorem 3.1. Let $X$ be a separable infinite-dimensional Banach space and $T$ $\in L(X)$. If $T$ satisfies the Frequent Hypercyclicity Criterion and if there exist an infinite-dimensional closed subspace $M_{0} \subset X$ and a set $A$ of positive lower density such that for any $x \in M_{0}$,

$$
T^{n} x \underset{n \rightarrow \infty}{\stackrel{n \in A}{\longrightarrow}} 0,
$$

then $T$ possesses a frequently hypercyclic subspace.

We can now state a sufficient condition for a frequently hypercyclic weighted shift on $l^{p}$ to possess a frequently hypercyclic subspace.

Theorem 3.2. Let $B_{w}: l^{p} \rightarrow l^{p}$ be a frequently hypercyclic weighted shift and

$$
G(k, C):=\left\{n \geq 0:\left\|B_{w}^{n} e_{k}\right\| \leq C\right\}, k \geq 0, C>0 .
$$

If there exist a strictly increasing sequence $\left(k_{l}\right)_{l \geq 1}$ of non-negative integers and $C>0$ such that $\bigcap_{l \geq 1} G\left(k_{l}, C\right)$ is of positive lower density, then $B_{w}$ possesses a frequently hypercyclic subspace.

Proof. Let $M_{0}=\overline{\operatorname{span}}\left\{e_{k_{l}}: l \geq 1\right\}$. If $x \in M_{0}$ and $n \in \bigcap_{l \geq 1} G\left(k_{l}, C\right)$, we have, by definition of $G(k, C)$,

$$
\left\|B_{w}^{n} x\right\|^{p}=\sum_{l=1}^{\infty}\left|x_{k_{l}}\right|^{p}\left\|B_{w}^{n} e_{k_{l}}\right\|^{p}=\sum_{k_{l} \geq n}\left|x_{k_{l}}\right|^{p}\left\|B_{w}^{n} e_{k_{l}}\right\|^{p} \leq \sum_{k_{l} \geq n} C^{p}\left|x_{k_{l}}\right|^{p} \underset{n \rightarrow \infty}{\longrightarrow} 0 .
$$

Since each frequently hypercyclic weighted shift $B_{w}$ on $l^{p}$ satisfies the Frequent Hypercyclicity Criterion and since $\bigcap_{l \geq 1} G\left(k_{l}, C\right)$ is of positive lower density, we conclude by using Theorem 3.1 
Example 3.3 (Weighted shift with a frequently hypercyclic subspace). Let $w=$ $\left(4,1^{a_{1}}, 4,1^{a_{2}}, 4,1^{a_{3}}, 4,1^{a_{4}}, 4, \ldots\right)$ where $\left(a_{n}\right)$ is a sequence of positive integers satisfying $a_{n+1} \geq \sum_{k=1}^{n} a_{k}+(n+1)$ and $1^{a_{n}}$ designates a block of 1 of length $a_{n}$. For any value of $a_{n}$, the weighted shift $B_{w}$ is an operator on $l^{p}$, and by (2.3),$B_{w}$ is frequently hypercyclic if and only if

$$
\sum_{k=1}^{\infty} \frac{a_{k}}{\left(4^{k}\right)^{p}}<\infty
$$

Moreover, we see that

$$
G\left(\sum_{k=1}^{n} a_{k}+n, 1\right)=\left[0, a_{n}\right] \cup\left[\sum_{k=1}^{n} a_{k}+(n+1), \infty[,\right.
$$

and since $\left(a_{n}\right)$ satisfies $a_{n+1} \geq \sum_{k=1}^{n} a_{k}+(n+1)$, we get

$$
\begin{aligned}
A:=\bigcap_{n=1}^{\infty} G\left(\sum_{k=1}^{n} a_{k}+n, 1\right) & =\bigcap_{n=1}^{\infty}\left([ 0 , a _ { n } ] \cup \left[\sum_{k=1}^{n} a_{k}+(n+1), \infty[)\right.\right. \\
& =\left[0, a_{1}\right] \cup \bigcup_{n=1}^{\infty}\left[\sum_{k=1}^{n} a_{k}+(n+1), a_{n+1}\right] .
\end{aligned}
$$

In particular, we deduce that if

$$
\liminf _{n} \frac{a_{n}-\sum_{k=1}^{n-1} a_{k}-n}{\sum_{k=1}^{n} a_{k}+(n+1)}>0
$$

then $A$ is a set of positive lower density. We remark that, if we let $a_{n}=3^{n}$, then $a_{n+1} \geq \sum_{k=1}^{n} a_{k}+(n+1)$ because

$$
\sum_{k=1}^{n} 3^{k}+(n+1)=\frac{3}{2}\left(3^{n}-1\right)+(n+1) \leq \frac{3^{n+1}}{2}+(n+1) \leq 3^{n+1} .
$$

Moreover, if $a_{n}=3^{n}$, then (3.1) and (3.2) are satisfied since we have

$$
\begin{gathered}
\sum_{k=1}^{\infty} \frac{3^{k}}{\left(4^{k}\right)^{p}}<\infty \text { and } \\
\liminf _{n} \frac{3^{n}-\sum_{k=1}^{n-1} 3^{k}-n}{\sum_{k=1}^{n} 3^{k}+(n+1)}=\liminf _{n} \frac{3^{n}-\frac{3}{2}\left(3^{n-1}-1\right)-n}{\frac{3}{2}\left(3^{n}-1\right)+(n+1)}=\frac{1}{3} .
\end{gathered}
$$

Therefore, we deduce from Theorem 3.2 that if $a_{n}=3^{n}$, then $B_{w}$ possesses a frequently hypercyclic subspace.

In view of Theorem 2.5 and Example 3.3. we deduce on the one hand that there exist some frequently hypercyclic weighted shifts on $l^{p}$ with a frequently hypercyclic subspace and some others without a frequently hypercyclic subspace, and, on the other hand, that the existence of a frequently hypercyclic subspace is not equivalent to the existence of a hypercyclic subspace and the frequent hypercyclicity. Therefore, we pose the following question:

Question. Which weighted shifts on $l^{p}$ possess a frequently hypercyclic subspace?

\section{ACKNOWLEDGMENT}

The author would like to thank the referee for valuable comments. 


\section{REFERENCES}

[1] Frédéric Bayart and Sophie Grivaux, Hypercyclicité: le rôle du spectre ponctuel unimodulaire (French, with English and French summaries), C. R. Math. Acad. Sci. Paris 338 (2004), no. 9, 703-708, DOI 10.1016/j.crma.2004.02.012. MR2065378 (2005c:47009)

[2] Frédéric Bayart and Sophie Grivaux, Frequently hypercyclic operators, Trans. Amer. Math. Soc. 358 (2006), no. 11, 5083-5117 (electronic), DOI 10.1090/S0002-9947-06-04019-0. MR2231886(2007e:47013)

[3] Frédéric Bayart and Étienne Matheron, Dynamics of linear operators, Cambridge Tracts in Mathematics, vol. 179, Cambridge University Press, Cambridge, 2009. MR 2533318 (2010m:47001)

[4] F. Bayart and I. Z. Ruzsa, Difference sets and frequently hypercyclic weighted shifts, Ergod. Th. Dynam. Sys. (2013), available on CJO2013. DOI 10.107/edts.2013.77.

[5] A. Bonilla and K.-G. Grosse-Erdmann, Frequently hypercyclic operators and vectors, Ergodic Theory Dynam. Systems 27 (2007), no. 2, 383-404, DOI 10.1017/S014338570600085X. MR2308137(2008c:47016)

[6] A. Bonilla and K.-G. Grosse-Erdmann, Frequently hypercyclic subspaces, Monatsh. Math. 168 (2012), no. 3-4, 305-320, DOI 10.1007/s00605-011-0369-2. MR2993952

[7] Karl-G. Grosse-Erdmann and Alfredo Peris Manguillot, Linear chaos, Universitext, Springer, London, 2011. MR2919812

[8] Manuel González, Fernando León-Saavedra, and Alfonso Montes-Rodríguez, Semi-Fredholm theory: hypercyclic and supercyclic subspaces, Proc. London Math. Soc. (3) 81 (2000), no. 1, 169-189, DOI 10.1112/S0024611500012454. MR1757050 (2001g:47013)

[9] Fernando León-Saavedra and Vladimír Müller, Hypercyclic sequences of operators, Studia Math. 175 (2006), no. 1, 1-18, DOI 10.4064/sm175-1-1. MR.2261697 (2007g:47012)

[10] Quentin Menet, Hypercyclic subspaces and weighted shifts, Adv. Math. 255 (2014), 305-337, DOI 10.1016/j.aim.2014.01.012. MR.3167484

Institut de Mathématique, Université de Mons, 20 Place du Parc, 7000 Mons, BelGIQUE

E-mail address: Quentin.Menet@umons.ac.be 